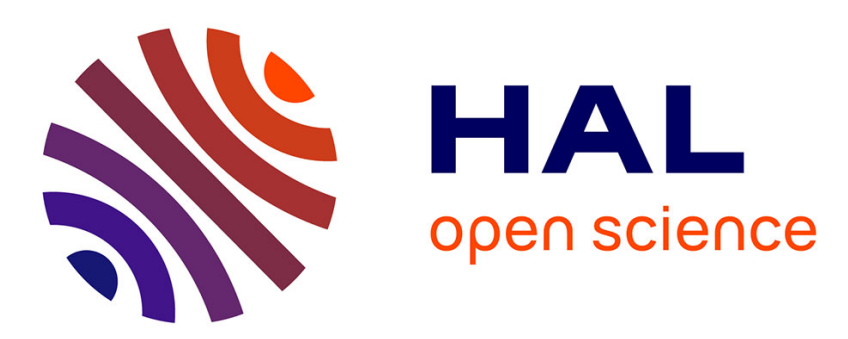

\title{
Principe de nouvelles mesures sur les écoulements par échauffements optiques localisés
}

\author{
P.-G. de Gennes
}

\section{To cite this version:}

P.-G. de Gennes. Principe de nouvelles mesures sur les écoulements par échauffements optiques localisés. Journal de Physique Lettres, 1977, 38 (1), pp.1-3. 10.1051/jphyslet:019770038010100 jpa00231312

\section{HAL Id: jpa-00231312 https://hal.science/jpa-00231312}

Submitted on 1 Jan 1977

HAL is a multi-disciplinary open access archive for the deposit and dissemination of scientific research documents, whether they are published or not. The documents may come from teaching and research institutions in France or abroad, or from public or private research centers.
L'archive ouverte pluridisciplinaire HAL, est destinée au dépôt et à la diffusion de documents scientifiques de niveau recherche, publiés ou non, émanant des établissements d'enseignement et de recherche français ou étrangers, des laboratoires publics ou privés. 


\title{
PRINCIPE DE NOUVELLES MESURES SUR LES ÉCOULEMENTS PAR ÉCHAUFFEMENTS OPTIQUES LOCALISÉS
}

\author{
P. G. DE GENNES
}

Collège de France 75231, Paris Cedex 05, France

(Reçu le 29 octobre 1976, accepté le 18 novembre 1976)

\begin{abstract}
Résumé. - Une distribution inhomogène de température (et donc d'indice) est créée au temps 0 dans un fluide par un flash optique. L'écoulement déplace et déforme cette distribution : on étudie la répartition au temps $t$ par diffraction d'une deuxième source lumineuse. Le diagramme de diffraction est insensible à une translation d'ensemble. Donc, on mesure directement des gradients de vitesse (en régime laminaire) ou, en régime turbulent, les fonctions de Richardson décrivant la séparation progressive de deux particules initialement voisines.

Ces fonctions doivent apporter une information beaucoup plus fine que les expériences de diffusion inélastique de la lumière par des particules en suspension.

Abstract. - An inhomogeneous distribution of temperature (and of refractive index) is created at $t=0$ in a fluid by an optical flash. Flow in the fluid displaces and distorts the distribution. We propose to study the pattern by diffraction of a second light source. The diffraction figure is unsensitive to an overall translation : thus one measures directly the gradients of the velocity field (in laminar flow) or, in a turbulent regime, the Richardson functions describing the progressive separation of two particles.

These measurements are thus very different and more powerful than the standard experiments based on the inelastic scattering of light by suspended particles.
\end{abstract}

Il existe peu de dispositifs permettant de mesurer localement les gradients de vitesse dans un écoulement. Nous proposons ici une méthode optique, inspirée par la méthode de Eichler et Pohl [1] pour la mesure des diffusivités thermiques.

A l'instant 0, par application d'un pulse lumineux, on crée dans le milieu (supposé légèrement absorbant) une distribution de température inhomogène

$$
T_{0}(\mathbf{r})=\bar{T}+\delta T_{0}(\mathbf{r}) \text {. }
$$

Il en résulte une modulation spatiale de l'indice

$$
\delta n_{0}(\mathbf{r})=\left(\frac{\partial n}{\partial T}\right)_{p} \delta T_{0}(\mathbf{r})
$$

Si on laisse maintenant passer un intervalle de temps $t$, la distribution est changée (a) par des mécanismes moléculaires de diffusion thermique, (b) par convection avec le champ de vitesses $\mathbf{v}(\mathbf{r}, \mathbf{t})$ présent dans le fluide. Dans tout ce qui suit, nous ne prendrons en compte que le processus (b); nous supposerons en outre que les vitesses induites par l'action de la pesanteur (sur un milieu légèrement inhomogène en densité) sont négligeables devant les vitesses intrinsèques de l'écoulement étudié.' Au bout du temps $t$, la distribution d'indice est devenue $\delta n(r, t)$ : on l'étudie par diffraction d'un faisceau de détection. Si $\mathbf{q}_{\mathbf{D}}$ est le vecteur de diffusion choisi, l'intensité diffractée est de la forme

$$
I_{t}=\int \mathrm{d} 3 \mathrm{~d} 4\langle\delta n(3 t) \delta n(4 t)\rangle \mathrm{e}^{i \mathbf{q}_{\mathrm{D}} \cdot\left(\mathbf{r}_{3}-\mathbf{r}_{4}\right)} D(3) D(4) .
$$

Le symbole $\langle>$ représente une moyenne à prendre quand l'écoulement est turbulent. La fonction $\dot{D}(\mathbf{r})$ est lentement variable et décrit l'étendue du faisceau de détection. Quand la convection intervient seule, on peut écrire

$$
I_{t}=\int \mathrm{d} 1 \mathrm{~d} 2 \mathrm{~d} 3 \mathrm{~d} 4 \delta n_{0}(1) \delta n_{0}(2) H_{t}(12 / 34) \mathrm{e}^{i \mathbf{q D}_{\mathrm{D}} \cdot\left(\mathbf{r}_{3}-\mathbf{r}_{4}\right)}
$$

La fonction $H_{t}(12 / 34)$ est la probabilité, pour une paire de particules du fluide, de passer des positions $(1,2)$ aux positions $(3,4)$ en un intervalle $t$.

Dans les applications envisagées ci-dessous, la source $\delta n_{0}(r)$ est, soit pratiquement ponctuelle (et créée par un flash bien focalisé), soit sinusoïdale $\delta n_{0} \simeq \cos \mathbf{q}_{\mathrm{s}} \cdot \mathbf{r} S(\mathbf{r})$, et créée par les "franges d'interférence d'un laser de puissance. (La fonction $S\left(r^{-1}\right)$ est une fonction enveloppe définissant la largeur $L_{\mathbf{S}}$ 
du domaine de franges) : on parlera respectivement de point chaud et de réseau chaud pour décrire ces deux types de conditions initiales.

1. RÉSEAU CHAUd EN RÉGIME LAMINAIRE : MESURE DES GRADIENTS DE VITESSE. - Si $L_{\mathrm{S}} \ll L$ et $t \ll t_{L}$ ou $L$ et $t_{L}$ sont les longueurs et les temps caractéristiques de l'écoulement macroscopique, l'effet de l'écoulement sur le réseau chaud est un effet de rotation et de cisaillement. On attend un pic de Bragg au vecteur d'onde

$$
\mathbf{q}_{\mathrm{D}}=(1+\hat{\mathbf{G}} t) \mathbf{q}_{\mathbf{S}} \quad(G t \ll 1)
$$

où $G_{i j}=\partial v_{i} / \partial x_{j}$ est le gradient. On peut envisager ici une méthode de Laue, dans laquelle le faisceau de détection est polychromatique, et où la direction de $\mathbf{q}_{\mathbf{D}}$ est seule déterminée - la tache de diffraction étant par exemple photographiée à l'instant $t$.

Si $\mathbf{q}_{s}$ est parallèle à l'axe (1), les angles de déflexion de $q_{\mathrm{D}}$ vers les axes (2) et (3) sont $t G_{21}$ et $t G_{31}$. En répétant la mesure pour plusieurs orientations de $\mathbf{q}_{\mathbf{s}}$ on aboutirait ainsi à une détermination complète du tenseur $\hat{\mathbf{G}}$.

2. PoINT CHAUd; RÉGIME TURBULENT : PREMIÈRE FONCTION DE RICHARDSON. - Si $\delta n_{0}(\mathbf{r})$ est très localisé (la zone de détection étant, elle, très grande) l'intensité diffractée devient

$$
I_{t}\left(\mathbf{q}_{\mathbf{D}}\right)=\text { Cte. } \int \mathrm{d} \boldsymbol{\rho} R_{t}(\boldsymbol{\rho}) \mathrm{e}^{i \mathbf{q}_{\mathbf{D} \cdot \boldsymbol{\rho}}}
$$

où $R_{t}(\boldsymbol{\rho})$ est la probabilité pour que 2 particules initialement contiguës se trouvent à la distance relative $\rho$ au temps $t$. Comme l'a souligné Richardson [2], la fonction $R_{t}$ est intéressante parce que sensible seulement aux tourbillons d'échelles inférieures à $\rho$ : l'expérience proposée ici est totalement différente des études classiques sur l'étalement d'un traceur ou d'un point chaud $[3,4]$, qui donnent une fonction de corrélation à une particule.

La mesure de $I_{t}\left(\mathbf{q}_{\mathrm{D}}\right)$ demande un faisceau de détection monochromatique, et en général un angle de diffraction faible - pour sonder $\boldsymbol{R}_{t}(\boldsymbol{\rho})$ à des distances $\rho \sim q_{\mathrm{D}}^{-1}$ qui soient dans le domaine inertial. Ainsi, pour une turbulence de grille, avec des espacements entre barreaux de $1 \mathrm{~mm}$, et une vitesse d'entrée $\bar{U}=10 \mathrm{~m} / \mathrm{s}$ (nombre de Reynolds dans l'eau $\sim 10^{4}$ ), les plus petits tourbillons sont d'environ $30 \mu$. La période $2 \pi / q_{\mathrm{D}}$ peut atteindre $300 \mu$, et est donc assez bien adaptée à l'observation du domaine inertial. Avec les hypothèses de Kolmogorov 1941 [5] la largeur spatiale $l(t)$ de la fonction $R_{t}(\rho)$ varierait dans ce domaine comme $l \sim \varepsilon^{1 / 2} t^{3 / 2}$ (où $\varepsilon$ est la dissipation par unité de masse).

Dans l'exemple ci-dessus les échelles temporelles associées aux différents tourbillons vont de $10^{-4}$ à $10^{-2} \mathrm{~s}$. Il faudrait donc enregistrer le spectre de diffraction dans un intervalle $(t, t+\Delta t)$ avec $\Delta t \lesssim 10^{-4} \mathrm{~s}$.
Le centre de la zone de détection doit être à une distance $\bar{U} t$ en aval de la source. Mais il faut en plus que la dimension $L_{\mathrm{D}}$ de la zone de détection soit grande devant la longueur de diffusion turbulente $\sqrt{v_{\text {eff }} t}$ de façon à ce que le point milieu de la paire étudié reste bien dans le champ. (Avec $t=10^{-3} \mathrm{~s}$ et un coefficient de transport turbulent $v_{\text {eff }} \sim 10 \mathrm{~cm}^{2} / \mathrm{s}$, $\left.\sqrt{v_{\text {eff }} t} \sim 1 \mathrm{~mm}\right)$.

3. RÉSEAU CHAUd EN RÉGIME TURBULENT $: 2^{\mathrm{e}}$ FONCTION DE RICHARDSON. - Ici le réseau initial (de période $2 \pi / q_{\mathrm{s}}$ ) est déformé de façon aléatoire par l'écoulement; il devient imparfait et aussi sa période moyenne change. La figure de diffraction, en fonction du vecteur d'onde de détection $\mathbf{q}_{\mathbf{D}}$, a la forme

$$
\begin{aligned}
I_{t}\left(\mathbf{q}_{\mathrm{S}} / \mathbf{q}_{\mathrm{D}}\right)=\int S(1) & S(2) H_{t}(12 / 34) D(1) D(2) \times \\
& \times \mathrm{e}^{i\left(\mathbf{q}_{\mathbf{D}} \cdot \mathbf{r}_{34}-\mathbf{q s} \cdot \mathbf{r}_{12}\right)} \mathrm{d} 1 \mathrm{~d} 2 \mathrm{~d} 3 \mathrm{~d} 4 .
\end{aligned}
$$

Si la turbulence est homogène $H_{t}$ ne dépend que de 3 vecteurs :

$$
\begin{gathered}
H_{t}(12 / 34)=K_{t}\left(\boldsymbol{\rho}_{0}, \boldsymbol{\rho}, \mathbf{X}\right) \\
\boldsymbol{\rho}_{0}=\mathbf{r}_{12}, \quad \boldsymbol{\rho}=\mathbf{r}_{34}, \quad \mathbf{X}=\frac{1}{2}\left(\mathbf{r}_{1}+\mathbf{r}_{2}-\mathbf{r}_{3}-\mathbf{r}_{4}\right)
\end{gathered}
$$

où $\mathbf{X}$ est le déplacement du point milieu. Supposons maintenant que $D(\mathbf{r})$ est constante (faisceau de détection large) : alors on peut intégrer l'éq. (5) sur $\mathbf{X}$ :

$I_{t}\left(\mathbf{q}_{\mathrm{S}} / \mathbf{q}_{\mathrm{D}}\right)=\mathrm{Cte} \int \mathrm{d} \boldsymbol{\rho}_{0} \mathrm{~d} \boldsymbol{\rho} R_{2 t}\left(\boldsymbol{\rho}_{\mathrm{o}} / \boldsymbol{\rho}\right) \mathrm{e}^{i\left(\mathbf{q}_{\mathrm{D}} \cdot \boldsymbol{\rho}-\mathbf{q}_{\mathrm{S}} \boldsymbol{\rho}_{0}\right)}$

La deuxième fonction de Richardson $R_{2 t}$ est définie par :

$$
R_{2 t}\left(\boldsymbol{\rho}_{0} / \boldsymbol{\rho}\right)=\int \mathrm{d} \mathbf{X} K_{t}\left(\boldsymbol{\rho}_{0}, \boldsymbol{\rho}, \mathbf{X}\right)
$$

Considérée comme fonction de $\rho$ (à $\rho_{0}$ et $t$ fixés) elle représente la loi de distribution de l'intervalle entre deux particules au temps $t$, sachant que cet intervalle était $\rho_{0}$ à l'instant 0 . La fonction $R_{2}$ contient une information assez riche sur les tourbillons, et mériterait d'être étudiée : ici encore il faudrait travailler avec des vecteurs $\mathbf{q}_{\mathrm{s}}$ et $\mathbf{q}_{\mathbf{D}}$ petits pour étudier le domaine inertial.

Notons enfin que la température n'est pas la seule variable utilisable pour modifier l'indice : avec des solutions de molécules photochromes, par exemple on peut avoir des dispositifs très sensibles [6]. Mais quel que soit le détail de l'excitation, on peut espérer mesurer des effets de séparation turbulente assez fins, et ceci plus simplement que dans les expériences d'échos de spin que nous avions proposées jadis [7]. 


\section{Bibliographie}

[1] Eichler, H., Salje, G., Stahl, H., J. Appl. Phys. 44 (1973) 5383. Poht, D., Schwarz, S., Irniger, V., Phys. Rev. Lett. 31 (1973) 32.

[2] Richardson, L., Proc. R. Soc. A 110 (1926) 709.

[3] Voir par exemple : Monin et YaGLom, Statistical fluid mechanics, Vol. II (MIT press) 1975.

Bradshaw, P., An introduction to turbulence (Pergamon) 1971.
[4] Les mesures par diffusion de lumière sur des particules en suspension déterminent seulement la loi de répartition des vitesses en écoulement turbulent, et ne sont pas sensibles aux effets locaux discutés ici. Voir par exemple BECKER, $H$. et al., J. Fluid. Mech. 30 (1967) 259.

[5] Kolmogorov, A., C.R.A.S. (URSS) 30 (1941) 301, 32, 16.

[6] RoNDELEZ, F., à paraître.

[7] De Gennes, P. G., Phys. Rev. Lett. 29A (1969) 20. 